\title{
Creation of partial band gaps in anisotropic photonic-band-gap structures
}

\author{
Zhi-Yuan Li \\ Department of Physics, The University of Hong Kong, Pokfulam Road, Hong Kong, China \\ and Institute of Physics, Chinese Academy of Sciences, P.O. Box 603, Beijing 100080, Beijing, China \\ Jian Wang \\ Department of Physics, The University of Hong Kong, Pokfulam Road, Hong Kong, China \\ Ben-Yuan Gu \\ Institute of Physics, Chinese Academy of Sciences, P.O. Box 603, Beijing 100080, Beijing, China
}

(Received 2 March 1998)

\begin{abstract}
The photonic-band-gap (PBG) structure composed of an anisotropic-dielectric sphere in uniform dielectric medium is studied by solving Maxwell's equations using the plane-wave expansion method. In particular, for a uniaxial material with large principal refractive indices and sufficient anisotropy between them, the photonic band structures possess a full band gap in the whole Brillouin zone for a diamond lattice. Furthermore, in the $1 / 3$ partial Brillouin zone where the Bloch wave vector has a dominant component along the extraordinary axis of uniaxial sphere, the photonic band structures are found to exhibit full band gaps for all the other lattices such as face-centered-cubic, body-centered-cubic, and simple-cubic lattices, although a complete band gap does not open in the whole Brillouin zone. The partial band gaps persist at a very low filling fraction of uniaxial sphere. This phenomenon is attributed to the breakdown of the photonic band degeneracy at high-symmetry points of the Brillouin zone by the anisotropy of material dielectricity. The combination of such an anisotropic PBG structure with the self-arrangement technique of colloidal crystal may provide a possible way to fabricate the three-dimensional photonic crystal in visible and infrared regimes. The application of a strong electric field may bring into alignment the extraordinary axis of uniaxial sphere as this configuration of spheres is most favorable thermodynamically. [S0163-1829(98)06231-6]
\end{abstract}

\section{INTRODUCTION}

Since the pioneering work of Yablonovitch and John, ${ }^{1,2}$ there appears great interest in fabricating photonic-band-gap (PBG) structure in recent years. These structures exhibit "forbidden" frequency region where electromagnetic (EM) waves cannot propagate for both polarizations along any direction. This may bring about some peculiar physical phenomena, ${ }^{3-8}$ many of which are based on the suppression of the instantaneous emission in the presence of a photonic band gap. Furthermore, it is expected that PBG structures possess the possibility to be applied in several scientific and technical areas such as filters, optical switches, cavities, design of low-threshold lasers, etc. ${ }^{9,10}$ It is also proposed that such PBG structures may hold the key to the continued progress towards all-optical integrated circuits. ${ }^{11}$

It is well known that there exist only pseudogaps in an isotropic face-centered-cubic (fcc) lattice composed of a dielectric sphere in the uniform background medium because of symmetry-induced degeneracy between the conduction band and valence band at the $W$ point of the Brillouin zone. ${ }^{12,13}$ The diamond structure was then proposed to possess a full gap by theoretical simulations ${ }^{14}$ and was soon verified by experiments. ${ }^{15}$ The symmetry-induced degeneracy at the $W$ point is broken down by nonspherical atom configuration, and a complete band gap opens between the 2-3 photonic bands. Thereafter, quite a lot of nonspherical atom configurations of PBG structures were revealed to exhibit full band gaps. ${ }^{16-21}$ The fabrication of these intricate structures requires state-of-the-art microlithography techniques, such as electron-beam lithography and x-ray lithography. So far they are only surmounted in the microwave, millimeter, and submillimeter regimes. The ultimate goal in this field to fabricate a three-dimensional (3D) photonic crystal with a full band gap in the optical regimes remains a challenge due to the complexity of microfabrication technique in micrometer- or submicrometer-size regimes.

Similar to the introduction of asymmetric atomic configuration into the PBG structure, another possible way to break down the degeneracy of photonic bands at high-symmetry points of the Brillouin zone would be asymmetry of material dielectricity, namely, material with anisotropic dielectric constant. Indeed, it has been demonstrated by Zabel and Stroud $^{22}$ that the anisotropy in the sphere dielectric function can split degenerate bands and this will narrow or even close band gaps for the diamond structure.

However, for an isotropic PBG structure with a spherical atom in simple lattices such as fcc, body-centered-cubic (bcc), and simple-cubic (sc) lattices, it is impossible to creat a full band gap due to degeneracy of bands. Thus the introduction of such an anisotropy of dielectric constant might instead lift the degeneracy of bands at the symmetry points and open up band gaps. Indeed we have found that for some uniaxial materials with large principal indices and sufficient anisotropy between them, the band gap can be opened in the 1/3 partial Brillouin zone for all the simple lattices, i.e., fcc, bcc, and sc lattices. In this partial zone the Bloch wave vector has the overwhelming component along the extraordinary 
axis of the uniaxial material. Although in general a complete band gap does not exist in the whole Brillouin zone, such a PBG structure is expected to allow an easier fabrication in the optical regime because it has a very simple lattice configuration and can be combined with the self-arrangement technique of the colloidal crystal. ${ }^{23-26}$ Furthermore, as it can forbid the propagation of EM waves in a wide range of directions, it will be of relevance in some applications.

In this paper, we first briefly introduce in Sec. II the plane-wave expansion method used in the calculations of photonic band structures for the anisotropic material. In Sec. III, we investigate in detail the photonic band structure of various lattices including the diamond, fcc, bcc, and sc lattices for a particular uniaxial material, Te (tellurium), which has large principal refractive indices and sufficient anisotropy between them. Then we discuss in Sec. IV the possibility of fabricating such an anisotropic PBG structure in micrometer- and submicrometer-size regimes. Finally summary and conclusions are given in Sec. V.

\section{MODEL AND FORMALISM}

Nature offers a lot of anisotropic materials that are lossless and transparent in the visible or infrared regime, among whom are uniaxial crystal and biaxial crystal. The uniaxial material has two different principal-refractive indices known as ordinary-refractive index $n_{o}$ and extraordinary-refractive index $n_{e}$, while the biaxial crystal has three different principal refractive indices, namely, $n_{x} \neq n_{y} \neq n_{z}$. For such anisotropic materials, the dielectric constant $\epsilon$ is a dyadic (second rank tensor). In the principal coordinates, the diagonal elements of $\epsilon$ are related to the principal-refractive indices as

$$
\epsilon_{x x}=n_{x}^{2}, \quad \epsilon_{y y}=n_{y}^{2}, \quad \epsilon_{z z}=n_{z}^{2},
$$

while other dyadic elements are all zero.

In a periodic anisotropic structure where the dielectric constant $\epsilon(\mathbf{r})$ is a dyadic and position dependent, Maxwell's equations for EM waves can be written as

$$
\begin{gathered}
\nabla \times \mathbf{E}=i(\omega / c) \mathbf{H}, \quad \nabla \times \mathbf{H}=-i(\omega / c) \mathbf{D} . \\
\nabla \cdot \mathbf{H}=0, \quad \nabla \cdot \mathbf{D}=0 .
\end{gathered}
$$

The electric displacement vector $\mathbf{D}$ is related to the electric field $\mathbf{E}$ as

$$
\mathbf{D}(\mathbf{r})=\epsilon(\mathbf{r}) \cdot \mathbf{E}(\mathbf{r})
$$

for anisotropic materials. We adopt the method of Ho, Chan, and Soukoulis ${ }^{14}$ to solve photonic band structures of such an anisotropic periodic structure. In this way, Maxwell's equations can be further simplified to an equation satisfied by the magnetic field $\mathbf{H}$ as

$$
\nabla \times\left[\epsilon^{-1}(\mathbf{r}) \cdot(\nabla \times \mathbf{H})\right]=\frac{\omega^{2}}{c^{2}} \mathbf{H},
$$

where $\epsilon^{-1}(\mathbf{r})$ is the inverse dyadic of $\epsilon(\mathbf{r})$. Since $\epsilon(\mathbf{r})$ is periodic, we can use Bloch's theorem to expand the $\mathbf{H}$ field in terms of plane waves,

$$
\mathbf{H}(\mathbf{r})=\sum_{\mathbf{G}} \sum_{\lambda=1}^{2} h_{\mathbf{G}, \lambda} \hat{\mathbf{e}}_{\lambda} e^{i(\mathbf{k}+\mathbf{G}) \cdot \mathbf{r}},
$$

where $\mathbf{k}$ is a wave vector in the Brillouin zone of the lattice, $\mathbf{G}$ is a reciprocal-lattice vector, and $\hat{\mathbf{e}}_{1}, \hat{\mathbf{e}}_{2}$ are orthogonal unit vectors that are both perpendicular to wave vector $\mathbf{k}+\mathbf{G}$ because of the transverse character of magnetic field $\mathbf{H}$ (i.e., $\nabla \cdot \mathbf{H}=0)$. The dielectric constant dyadic can also be expanded into its Fourier form as

$$
\epsilon^{-1}(\mathbf{r})=\sum_{\mathbf{G}} \epsilon^{-1}(\mathbf{G}) e^{i \mathbf{G} \cdot \mathbf{r}}
$$

where the Fourier transform coefficient $\epsilon^{-1}(\mathbf{G})$ is also a dyadic and can be obtained either analytically or numerically, quite the same way as in the case of an isotropic PBG structure.

Substituting Eq. (4) and Eq. (5) into Eq. (3), we obtain the following linear matrix equations:

$$
\sum_{\mathbf{G}^{\prime}, \lambda^{\prime}} H_{\mathbf{G}, \mathbf{G}^{\prime}}^{\lambda, \lambda^{\prime}} h_{\mathbf{G}^{\prime}, \lambda^{\prime}}=\frac{\omega^{2}}{c^{2}} h_{\mathbf{G}, \lambda},
$$

where

$$
\begin{aligned}
H_{\mathbf{G}, \mathbf{G}^{\prime}}^{\lambda, \lambda^{\prime}}=|\mathbf{k}+\mathbf{G}| & \left|\mathbf{k}+\mathbf{G}^{\prime}\right| \\
& \times\left(\begin{array}{cc}
\hat{\mathbf{e}}_{2} \cdot \epsilon_{\mathbf{G}, \mathbf{G}^{\prime}}^{-1} \cdot \hat{\mathbf{e}}_{2}, & -\hat{\mathbf{e}}_{2} \cdot \boldsymbol{\epsilon}_{\mathbf{G}, \mathbf{G}^{\prime}}^{-1} \cdot \hat{\mathbf{e}}_{1^{\prime}} \\
-\hat{\mathbf{e}}_{1} \cdot \epsilon_{\mathbf{G}, \mathbf{G}^{\prime}}^{-1} \cdot \hat{\mathbf{e}}_{2^{\prime}} & \hat{\mathbf{e}}_{1} \cdot \boldsymbol{\epsilon}_{\mathbf{G}, \mathbf{G}^{\prime}}^{-1} \cdot \hat{\mathbf{e}}_{1^{\prime}}
\end{array}\right)
\end{aligned}
$$

with $\epsilon_{\mathbf{G}, \mathbf{G}^{\prime}}^{-1}=\epsilon^{-1}\left(\mathbf{G}-\mathbf{G}^{\prime}\right)$.

The matrix $H_{\mathbf{G}, \mathbf{G}^{\prime}}^{\lambda, \lambda^{\prime}}$ is real and symmetric; thus the dispersion relation of EM waves can be solved by using standard matrix diagonalization techniques for the linear system of Eq. (6). In our calculations, the convergence is quite fast for low-energy bands. For the fcc, bcc, and sc lattices, we adopt 343 plane waves for each polarization of the magnetic field, while for the diamond structure, we adopt 512 plane waves. The convergence accuracy is better than $1 \%$ for the lowest ten energy bands.

Because of anisotropy of dielectric constant, Maxwell's equations do not conserve in some symmetry transform operations of the lattice. The reduction of crystal symmetry results in the inequivalence of the photonic band structures along previously equivalent directions in the Brillouin zone. To demonstrate the existence of a complete band gap for such an anisotropic PBG structure, one has to calculate the photonic band structure along high-symmetry lines in various regions of the Brillouin zone. Another equivalent way is to investigate the photonic band structure along the symmetry lines in a fixed region of the Brillouin zone, while transforming the dielectric constant dyadic in real space. ${ }^{22}$ In this paper we would like to adopt the latter method to treat the anisotropic PBG structures in the diamond, fcc, bcc, and sc lattices. 


\section{ANISOTROPIC PHOTONIC-BAND STRUCTURES IN VARIOUS LATTICES}

For an isotropic PBG structure, the refractive index of atom must be sufficiently large so that the resonant scattering of EM waves is prominent enough to open a band gap. This mechanism should also be applicable to an anisotropic PBG structure. Furthermore, in order to lift the degeneracy of bands at high-symmetry points of the Brillouin zone remarkably enough to allow the opening of a band gap, the atomic dielectric constant should have sufficient anisotropy. This is indeed the case according to our numerical simulations. We have tried a lot of anisotropic materials in the fcc lattice, which has a Brillouin zone closest to a sphere and thus is most likely to exhibit a full photonic band gap. We found that the symmetry at the $W$ point was broken down even in the case of weak anisotropy of material dielectricity. However, for most of them the band gap is not opened either because of low refractive index or due to insufficient anisotropy. Only a few materials known in reality can match these two severe conditions. ${ }^{27}$ Thus, we focus our concentration on those anisotropic materials with large principal indices and sufficient anisotropy between them.

In particular, we study the photonic-band structure of an anisotropic material, Te (tellurium), which is a kind of positive uniaxial crystals with principal-refractive indices $n_{e}$ $=6.2$ and $n_{o}=4.8$.

The PBG structure with so large principal indices should exhibit full band gaps in the diamond structure, as is the case for the isotropic PBG structure. Although it has been demonstrated that anisotropy of atom dielectricity would narrow or even close band gaps due to the splitting of photonic bands, ${ }^{22}$ it is expected that such a splitting will not be so severe as to close the band gaps in the diamond structure for this uniaxial material. To investigate the existence of photonic band gaps for such a uniaxial material, we calculate the photonic-band structures along the symmetry lines in a fixed partial region of the Brillouin zone (a fcc Brillouin zone for the diamond structure). The high-symmetry points of this partial Brillouin zone have the coordinates as $\Gamma=(0,0,0)$, $X=(2 \pi / a)(1,0,0), W=(2 \pi / a)\left(1, \frac{1}{2}, 0\right), K=(2 \pi / a)\left(\frac{3}{4}, \frac{3}{4}, 0\right)$, $L=(2 \pi / a)\left(\frac{1}{2}, \frac{1}{2}, \frac{1}{2}\right)$, and $U=(2 \pi / a)\left(1, \frac{1}{4}, \frac{1}{4}\right)$. Here $a$ is the cubic constant of a diamond lattice. The standard Brillouin zone of various lattices can be found in many textbooks and thus is not shown here; one can refer to Ref. 28. It is evident that we have chosen a partial Brillouin zone in which the Bloch wave vector dominates along the $x$-axis direction.

The photonic band structure in the whole Brillouin zone can well be denoted by transforming the dielectric constant dyadic. For uniaxial material, the dielectric dyadic has only three cases for diagonal element permutation as (a) $n_{x}$ $=n_{e}, n_{y}=n_{z}=n_{o}$; (b) $n_{y}=n_{e}, n_{x}=n_{z}=n_{o}$; and (c) $n_{z}$ $=n_{e}, n_{x}=n_{y}=n_{o}$. Each case just corresponds to one-third of the Brillouin zone. The corresponding calculated photonic band structures for a diamond PBG structure consisting of a Te (uniaxial) sphere in air background with a filling fraction of $f=30 \%$ are displayed in Figs. 1(a), 1(b), and 1(c), respectively. It is evident that for each $1 / 3$ partial Brillouin zone there still exists a remarkable band gap between 2-3 bands irrespective of the band splitting. So a true band gap opens in the whole Brillouin zone for this anisotropic PBG structure.

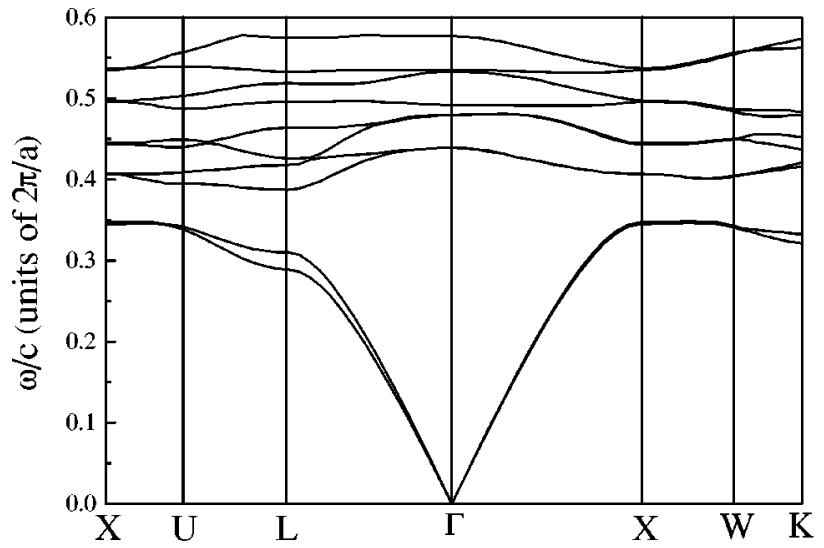

(a)

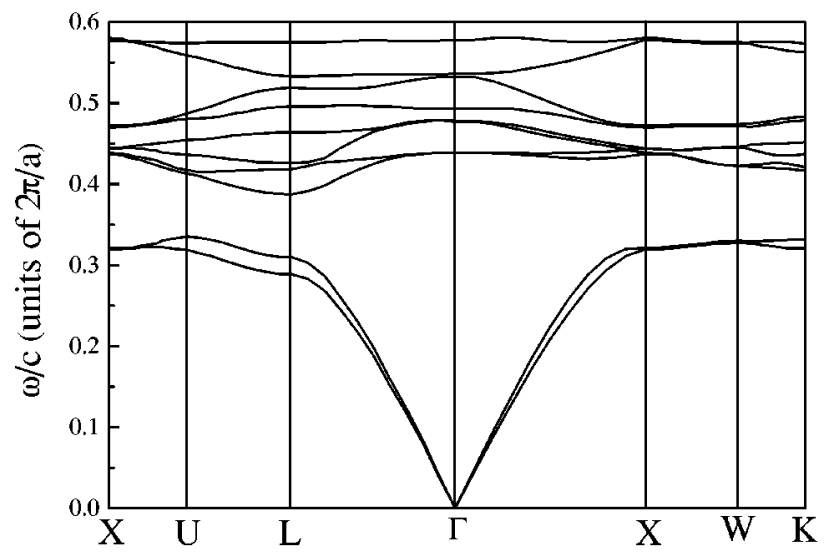

(b)

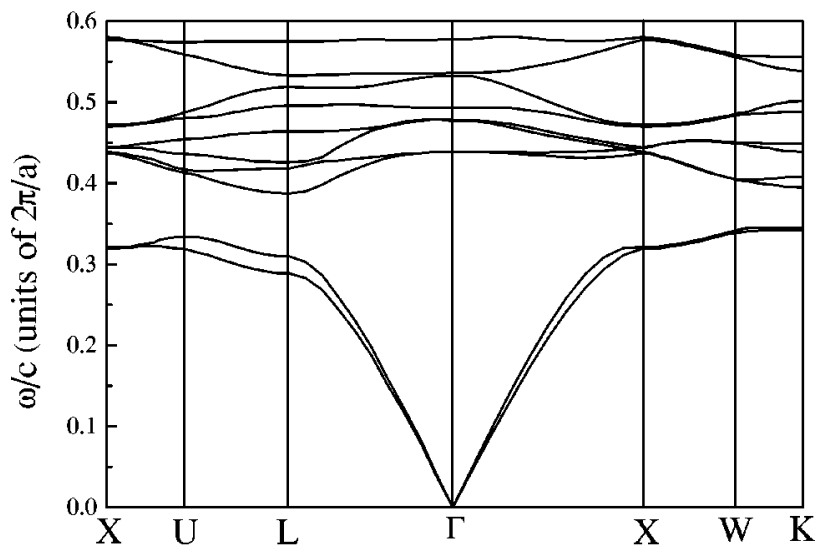

(c)

FIG. 1. Calculated anisotropic photonic band structure corresponding to three inequivalent $1 / 3$ partial Brillouin zones of a diamond lattice as (a) $n_{x}=n_{e}, n_{y}=n_{z}=n_{o}$; (b) $n_{y}=n_{e}, n_{x}=n_{z}=n_{o}$; and (c) $n_{z}=n_{e}, n_{x}=n_{y}=n_{o}$, respectively. The high-symmetry points are chosen as $\Gamma=(0,0,0), X=(2 \pi / a)(1,0,0), \quad W=(2 \pi /$ a) $\left(1, \frac{1}{2}, 0\right), \quad K=(2 \pi / a)\left(\frac{3}{4}, \frac{3}{4}, 0\right), \quad L=(2 \pi / a)\left(\frac{1}{2}, \frac{1}{2}, \frac{1}{2}\right), \quad$ and $\quad U$ $=(2 \pi / a)\left(1, \frac{1}{4}, \frac{1}{4}\right)$, where $a$ is the cubic lattice constant of a diamond lattice. The PBG structure is composed of a Te (uniaxial) sphere in air background. The uniaxial sphere has a filling fraction of $f=30 \%$ and principal refractive indices of $n_{e}=6.2$ and $n_{o}=4.8$.

The band gap to midgap ratios $\left(\Delta \omega / \omega_{g}\right)$ are $10.3 \%, 14.4 \%$, and $11.8 \%$, respectively; all of them are less than the corresponding ratio of isotropic PBG structure, which is about 


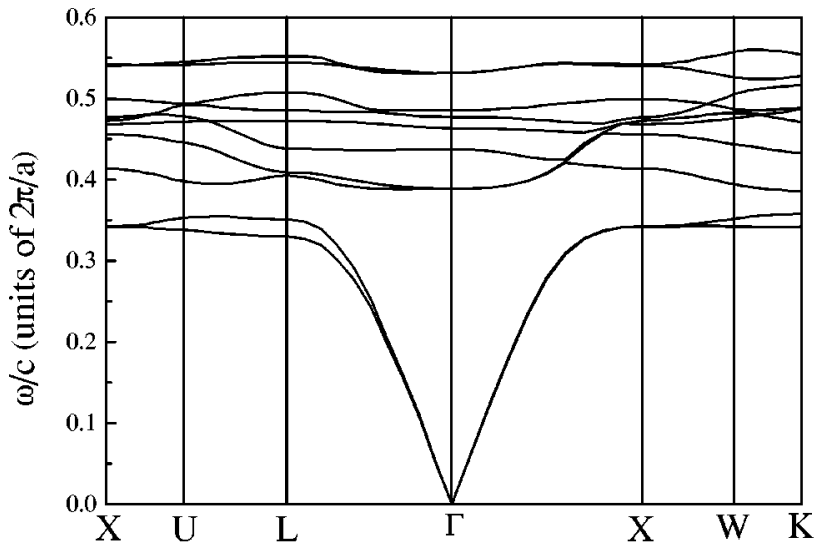

(a)

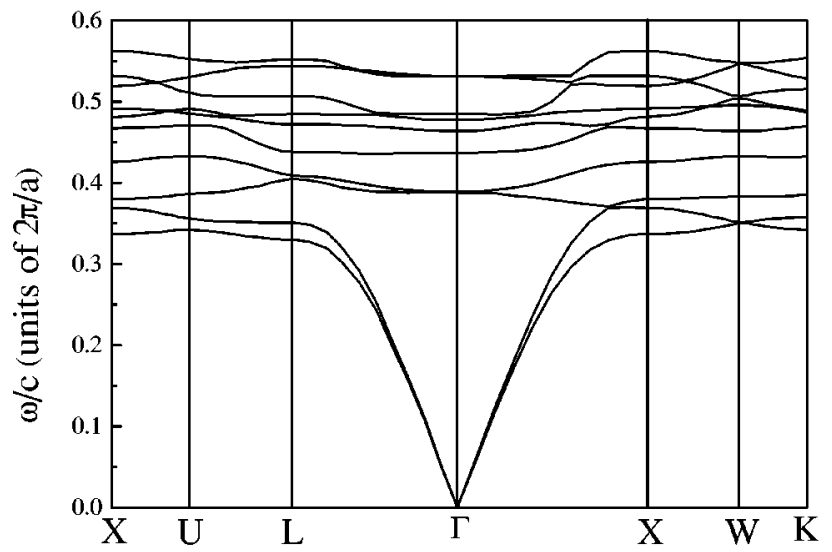

(b)

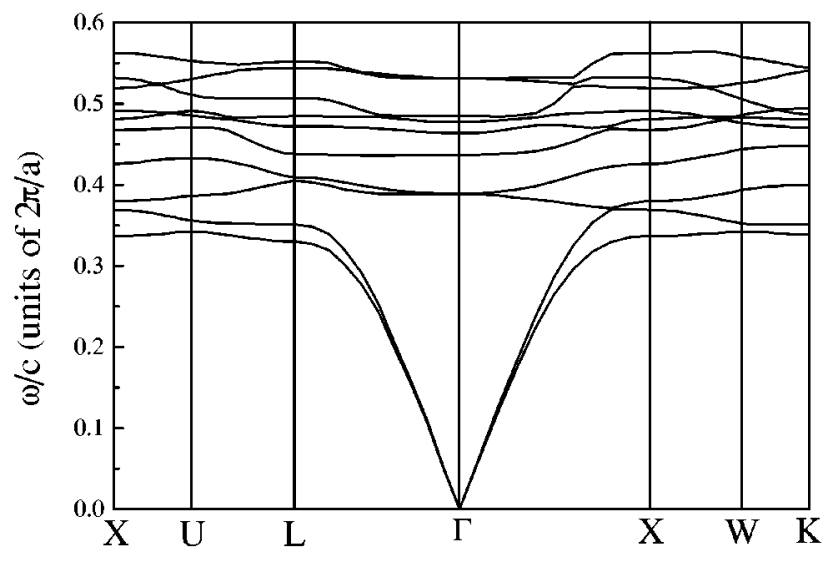

(c)

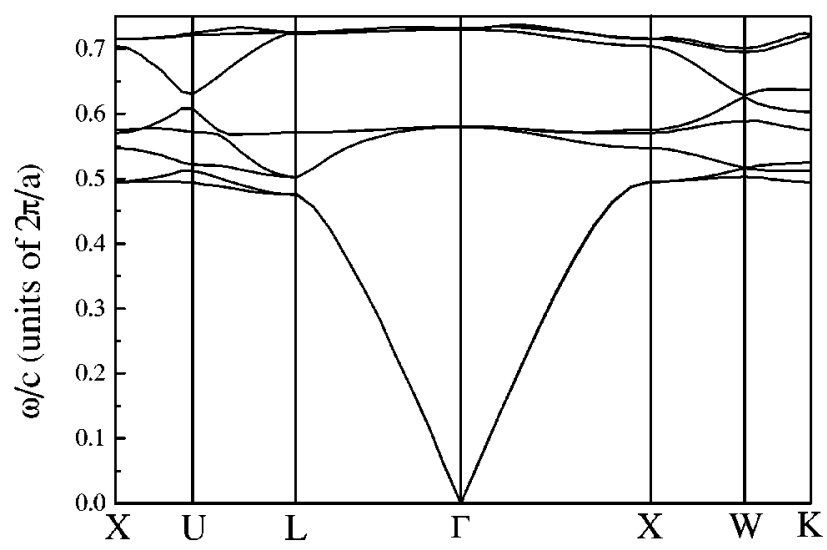

(d)

FIG. 2. Calculated anisotropic photonic band structure corresponding to three inequivalent $1 / 3$ partial Brillouin zones of a fcc lattice as (a) $n_{x}=n_{e}, n_{y}=n_{z}=n_{o}$; (b) $n_{y}=n_{e}, n_{x}=n_{z}=n_{o}$; and (c) $n_{z}=n_{e}, n_{x}=n_{y}=n_{o}$, respectively. The high-symmetry points are chosen the same as in Fig. 1. The anisotropic PBG structure consists of a Te sphere in air background with a filling ratio of $f=30 \%$. (d) The photonic band structure for an isotropic fcc lattice with a refractive index contrast of $n=3.6$ and a filling fraction of $f=30 \%$. $a$ is the cubic lattice constant of a fcc lattice.

$16.5 \%$ for a refractive index contrast of $n=5.3$ and a filling fraction of $f=30 \%$ in the diamond structure. This narrowing of band gap is due to band splitting caused by the reduction of crystal symmetry by anisotropic dielectricity. One can compare Figs. 1(a)-1(c) with the photonic band structure of an isotropic diamond PBG structure (see Ref. 14). The band splitting is most prominent at the $L$ point for the 1-2 and 3-4 photonic bands, and this narrows the band gap remarkably.

The introduction of dielectric anisotropy into the PBG structure can lift degeneracy of bands at high-symmetry points of the Brillouin zone, and this narrows the overall band gap of the diamond structure. Yet, for other simpler lattices such as fcc, bcc, and sc lattices, in which the isotropic PBG structures are well known to forbid complete band gap because of band degeneracy at some high-symmetry points, which are $W$ point for a fcc lattice, $H$ and $P$ points for a bcc lattice, and $M$ and $R$ points for a sc lattice, the sufficient breakdown of this symmetry-induced degeneracy by anisotropy of atom dielectricity may instead provide a possibility to open the band gaps.

To investigate the photonic band structure of an anisotropic fcc PBG structure, we choose the same fixed partial Brillouin zone as in the diamond lattice. The photonic band structures corresponding to three inequivalent $1 / 3$ Brillouin zone (a) $n_{x}=n_{e}, n_{y}=n_{z}=n_{o}$; (b) $n_{y}=n_{e}, n_{x}=n_{z}=n_{o}$; and (c) $n_{z}=n_{e}, n_{x}=n_{y}=n_{o}$ are displayed in Figs. 2(a), 2(b), and 2(c), respectively, for the uniaxial material Te. The fcc PBG structure is consisting of a Te sphere in air background with a filling fraction of $f=30 \%$. As a comparison, we display in Fig. 2(d) the photonic band structure of an isotropic fec PBG structure with a refractive index contrast of $n=3.6$ between the sphere and the background medium and a filling fraction of $f=30 \%$.

One can see that, generally, the degeneracy of 2-3 photonic bands at the $W$ point is lifted by anisotropy of material dielectricity. Furthermore, the third band is lifted sufficiently along the $X-K$ line and thus it provides a possible way to create a band gap. However, only in the $1 / 3$ partial Brillouin zone with the wave vector dominating along the extraordinary axis (namely, $n_{x}=n_{e}, n_{y}=n_{z}=n_{o}$ ), there exists a band gap with a width of $\Delta \omega / \omega_{g}=7.3 \%$ [see Fig. 2(a)]. In two other $1 / 3$ partial Brillouin zones, the anisotropy induced splitting of the lowest two bands is so remarkable that the 2-3 bands close together at somewhere along the $\Gamma-X$ line [see Figs. 2(b) and 2(c)]. Thus, a full band gap does not exist in the whole Brillouin zone. Instead, it is rather a partial band gap opening in the $1 / 3$ partial Brillouin zone for such an anisotropic PBG structure. 


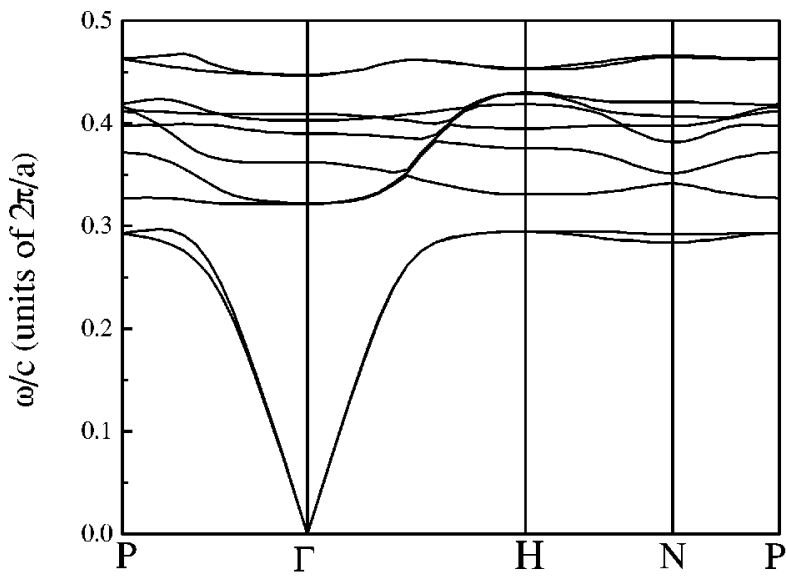

(a)

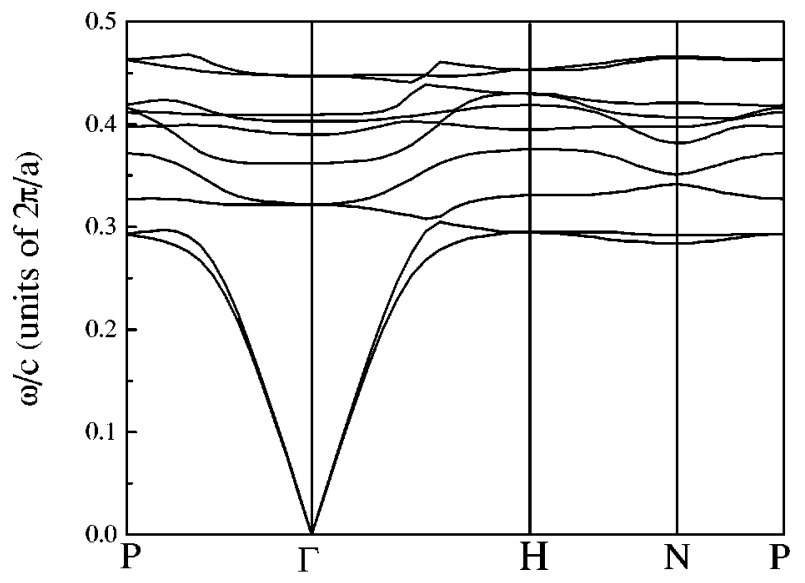

(b)

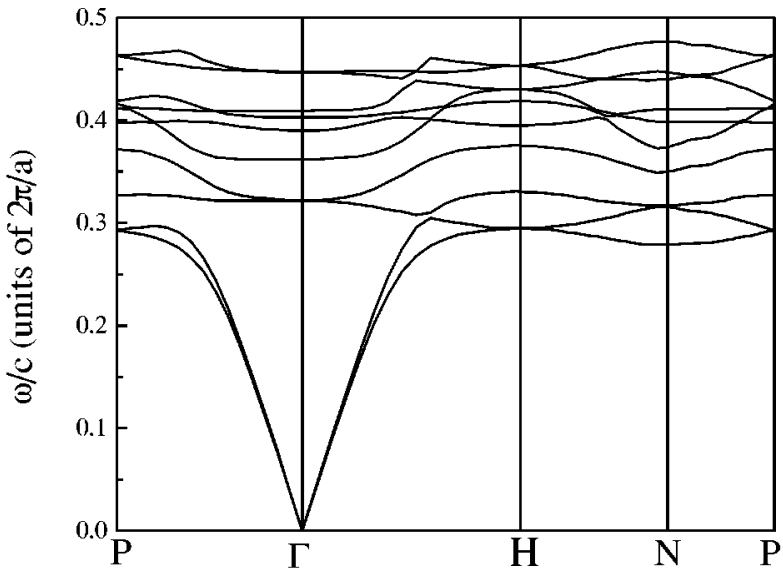

(c)

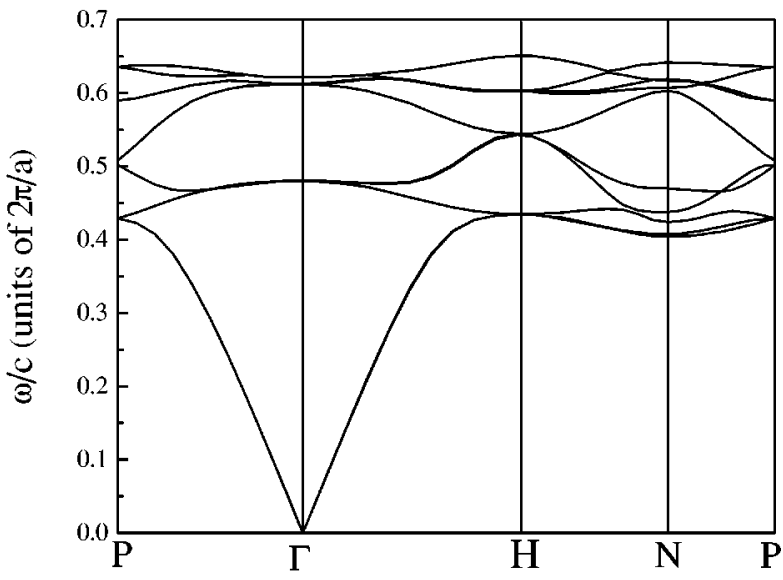

(d)

FIG. 3. Calculated anisotropic photonic band structure corresponding to three inequivalent $1 / 3$ partial Brillouin zones of a bcc lattice as (a) $n_{x}=n_{e}, n_{y}=n_{z}=n_{o}$; (b) $n_{y}=n_{e}, n_{x}=n_{z}=n_{o}$; and (c) $n_{z}=n_{e}, n_{x}=n_{y}=n_{o}$, respectively. The high-symmetry points are chosen as $\Gamma$ $=(0,0,0), H=(2 \pi / a)(1,0,0), N=(2 \pi / a)\left(\frac{1}{2}, \frac{1}{2}, 0\right)$, and $P=(2 \pi / a)\left(\frac{1}{2}, \frac{1}{2}, \frac{1}{2}\right)$. $a$ is the cubic lattice constants of a bcc lattice. The bcc PBG structure consists of a Te sphere in air background with a filling fraction of $f=25 \%$. (d) The photonic band structure for an isotropic bcc lattice with a refractive index contrast of $n=3.6$ and a filling fraction of $f=25 \%$.

The sufficient anisotropy-induced band splitting can open a partial band gap when light propagates dominantly along the extraordinary axis of the uniaxial sphere. Therefore, it is expected that, provided the presence of a sufficient anisotropy of atom dielectricity, such a band splitting may be large enough to open a band gap near high-symmetry points for the bcc and sc lattices. These two lattices are usually regarded as poor candidates for a true band gap as their Brillouin zones are obviously deviated from a sphere, compared with the Brillouin zone of a fcc lattice.

Similar to the diamond and fcc lattices, for the bcc lattice we choose a fixed partial Brillouin zone in which the Bloch wave vector also dominates along the $x$-axis direction. In this zone the coordinates of some high-symmetry points read $\Gamma$ $=(0,0,0), H=(2 \pi / a)(1,0,0), N=(2 \pi / a)\left(\frac{1}{2}, \frac{1}{2}, 0\right)$, and $P$ $=(2 \pi / a)\left(\frac{1}{2}, \frac{1}{2}, \frac{1}{2}\right)$, where $a$ is the cubic constant of a bcc lattice. The photonic band structures along some important symmetry lines are displayed in Figs. 3(a), 3(b), and 3(c), respectively, corresponding to three inequivalent $1 / 3$ Brillouin zones as (a) $n_{x}=n_{e}, n_{y}=n_{z}=n_{o}$; (b) $n_{y}=n_{e}, n_{x}=n_{z}$ $=n_{o}$; and (c) $n_{z}=n_{e}, n_{x}=n_{y}=n_{o}$. The bcc PBG structure is consisting of a uniaxial Te sphere in air background with a filling fraction of $f=25 \%$. For the convenience of a comparison, the photonic band structure of an isotropic bcc PBG structure with a refractive index constant of $n=3.6$ and a filling fraction of $f=25 \%$ is shown in Fig. 3(d).

It is evident that degeneracy of the $2-3$ photonic bands at $H$ and $P$ points is lifted remarkably by the strong anisotropy of material dielectricity. Such a symmetry-induced degeneracy always lies in an isotropic PBG structure and results in the absence of a true band gap between the 2-3 bands [see Fig. 3(d)]. In the partial Brillouin zone with the Bloch wave vector overwhelming along the extraordinary axis, as shown in Fig. 3(a), a full band gap is created between the 2-3 bands with a gap width of $\Delta \omega / \omega_{g}=7.4 \%$. Another narrower band gap is created between the 8-9 bands with a gap width of $\Delta \omega / \omega_{g}=3.8 \%$. However, the band gap is closed in two other $1 / 3$ partial Brillouin zones due to the large band splitting of the lowest two bands along the $\Gamma-H$ symmetry line, as shown in Figs. 3(b) and 3(c). This is similar to the case in a fcc lattice as shown in Figs. 2(b) and 2(c).

The photonic band structures for a sc lattice are shown in Figs. 4(a), 4(b), and 4(c) along some important symmetry lines in the three inequivalent $1 / 3$ partial Brillouin zones as 


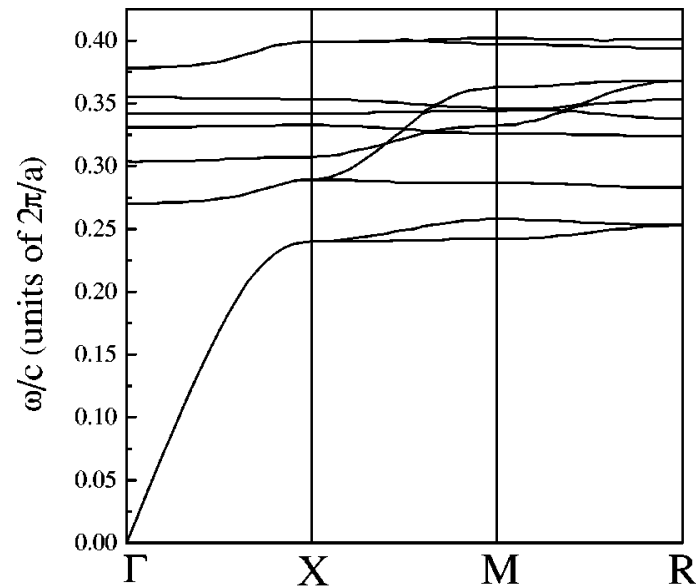

(a)

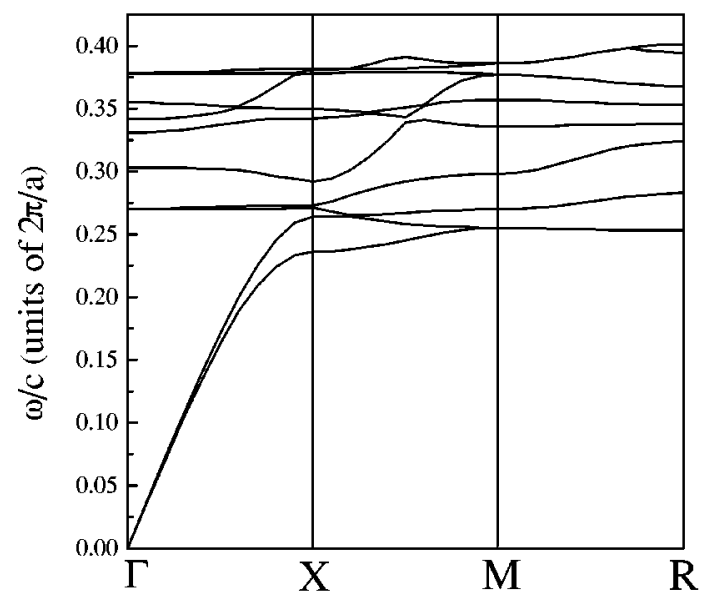

(b)

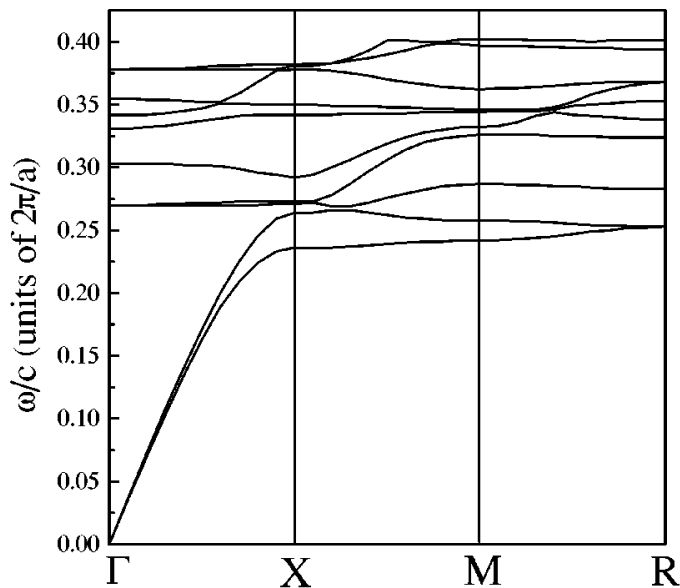

(c)

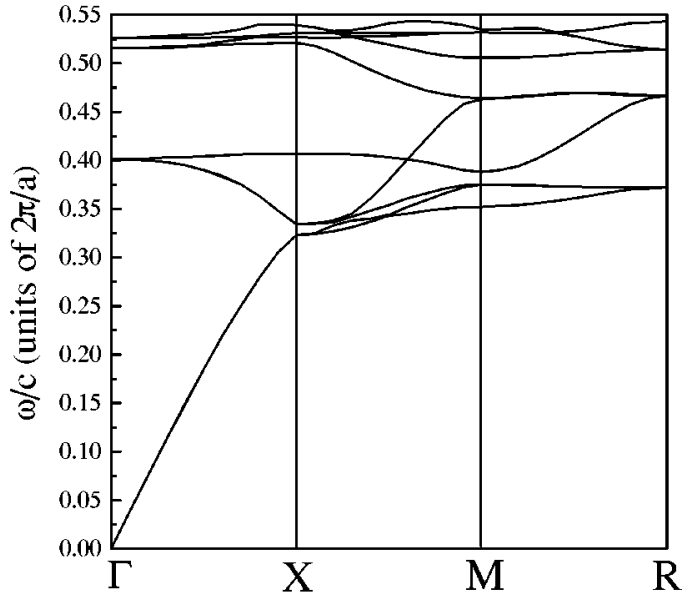

(d)

FIG. 4. Calculated anisotropic photonic band structure corresponding to three inequivalent $1 / 3$ partial Brillouin zones of a sc lattice as (a) $n_{x}=n_{e}, n_{y}=n_{z}=n_{o}$; (b) $n_{y}=n_{e}, n_{x}=n_{z}=n_{o}$; and (c) $n_{z}=n_{e}, n_{x}=n_{y}=n_{o}$, respectively. The high-symmetry points are chosen as $\Gamma$ $=(0,0,0), X=(\pi / a)(1,0,0), M=(\pi / a)(1,0,1)$, and $R=(\pi / a)(1,1,1) . a$ is the cubic lattice constant of a sc lattice. The sc PBG structure is composed of a Te sphere in air background with a filling fraction of $f=20 \%$. (d) The photonic band structure for an isotropic sc lattice with a refractive index contrast of $n=3.6$ and a filling fraction of $f=20 \%$.

(a) $n_{x}=n_{e}, n_{y}=n_{z}=n_{o} ;$ (b) $n_{y}=n_{e}, n_{x}=n_{z}=n_{o}$; and (c) $n_{z}=n_{e}, n_{x}=n_{y}=n_{o}$, respectively. The sc photonic crystal is composed of a Te sphere in air background and the filling fraction of sphere is $f=20 \%$. The fixed partial sc Brillouin zone is also chosen such that the Bloch wave vector dominates along the $x$-axis direction, and the coordinates of some high-symmetry points are $\Gamma=(0,0,0), X=(\pi / a)(1,0,0), M$ $=(\pi / a)(1,0,1)$, and $R=(\pi / a)(1,1,1)$, where $a$ is the sc lattice constant. We append in Fig. 4(d) the photonic band structure of an isotropic sc PBG with a refractive-index constant of $n=3.6$ and a filling fraction of $f=20 \%$, also for the convenience of comparison.

It is clearly seen that degeneracy of photonic bands at high-symmetry points $M$ and $R$ between the 2-3 bands does not allow the appearance of a true band gap for an isotropic PBG structure, as shown in Fig. 4(d). This degeneracy is destroyed by anisotropy of material dielectricity. Similar to the case in the fcc and bcc lattices, this anisotropy-induced band splitting results in the formation of a partial band gap between the 2-3 photonic bands and a second band gap between the $8-9$ bands in the $1 / 3$ partial Brillouin zone where the Bloch wave vector dominates along the extraordinary axis of the uniaxial sphere, as shown in Fig. $4(\mathrm{a}) . \Delta \omega / \omega_{g}$ of these two gaps are $4.6 \%$ and $2.6 \%$, respectively. Also similar to the fcc and bcc lattices, the band gap is closed in two other $1 / 3$ partial Brillouin zones due to a large splitting of the 1-2 bands near the $X$ point, as shown in Figs. 4(b) and 4(c).

Thus, one can find that for various lattices, a partial band gap can be formed by the sufficient anisotropy-induced band splitting, irrespective of remarkable differences among their Brillouin zone configurations. It seems that asymmetry of a PBG structure, either from atom configuration or from atom dielectricity, plays a more remarkable role in creating a full band gap than the configuration anisotropy of the Brillouin zone.

We have calculated anisotropic photonic band structures for several filling fractions of Te sphere in the fcc, bcc, and sc lattices, and found that the partial band gaps in the $1 / 3$ partial Brillouin zone along the extraordinary axis can persist in a very wide range of atom filling fractions. The plot of $\Delta \omega / \omega_{g}$ of the 2-3 band gap vs the filling fraction $f$ is displayed in Fig. 5 for anisotropic fcc PBG structure. The partial band gap can still be created at such a low filling fraction as $f=5 \%$. The maximum of $\Delta \omega / \omega_{g}$ is about $7.6 \%$ for $f$ $=27 \%$. 


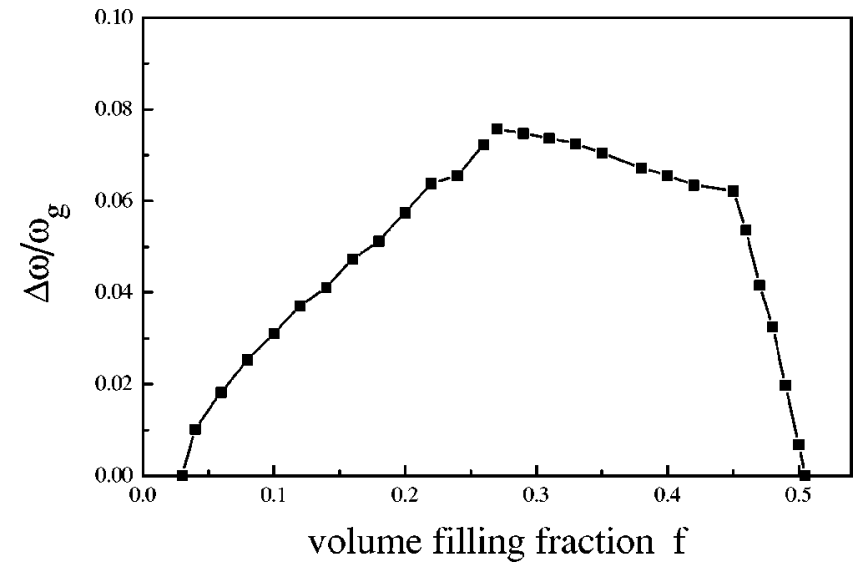

FIG. 5. Plots of $\Delta \omega / \omega_{g}$ of 2-3 partial band gap vs the filling fraction of Te sphere for a fcc lattice.

The dependences of the partial band-gap width on the filling fraction of Te sphere are displayed in Fig. 6 and Fig. 7 for the bcc and sc lattices, respectively. It is seen that both the 2-3 and 8-9 band gaps can also persist at a very low filling fraction of $f=5 \%$. For the 2-3 band gap, the maximum $\Delta \omega / \omega_{g}$ appears at the high- $f$ region, it is $9.6 \%$ for a bcc lattice at $f=30 \%$ and $5.7 \%$ for a sc lattice at $f=25 \%$. However the maximum $\Delta \omega / \omega_{g}$ of the 8-9 band gap lies in the low- $f$ region, for a bcc lattice it is $4.8 \%$ at $f=8 \%$, and for a sc lattice it is $4.0 \%$ at $f=9 \%$.

\section{SUGGESTIONS ON FABRICATION OF ANISOTROPIC PBG STRUCTURE}

First, one can see that such a uniaxial material Te exhibits quite favorable features in the photonic band structure. When light propagates dominantly along the extraordinary axis of the aligned spheres, it exhibits a full band gap for all the usual lattices in nature, namely, sc, bcc, fcc, and diamond lattices, irrespective of the large difference in configuration of their Brillouin zones. Second, the "atom" of the photonic crystal has a spherical shape, it is the simplest configuration in microfabrication. Furthermore, it provides the potential way to incorporate with the self-arrangement technique of colloidal crystal in the micrometer- and submicrometer-size

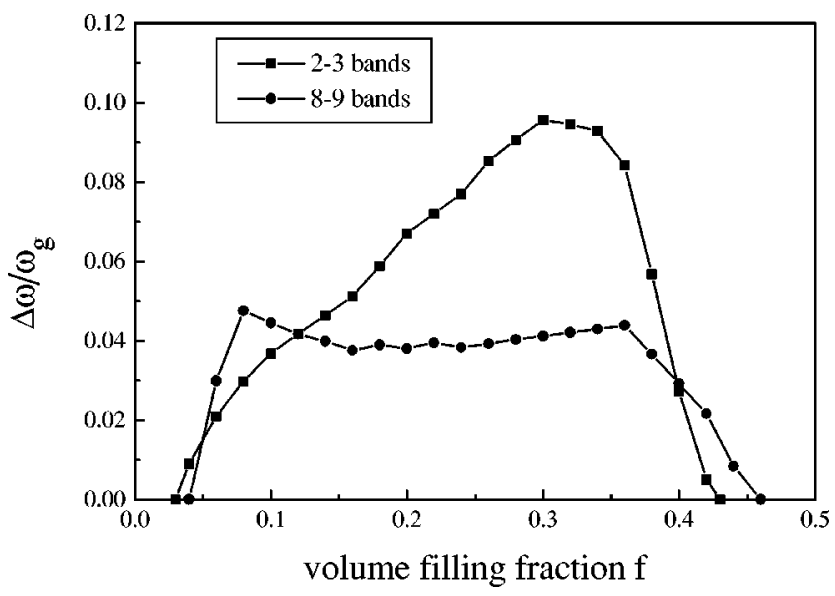

FIG. 6. Plots of $\Delta \omega / \omega_{g}$ of 2-3 and 8-9 partial band gaps vs filling fraction of Te sphere for a bcc lattice.

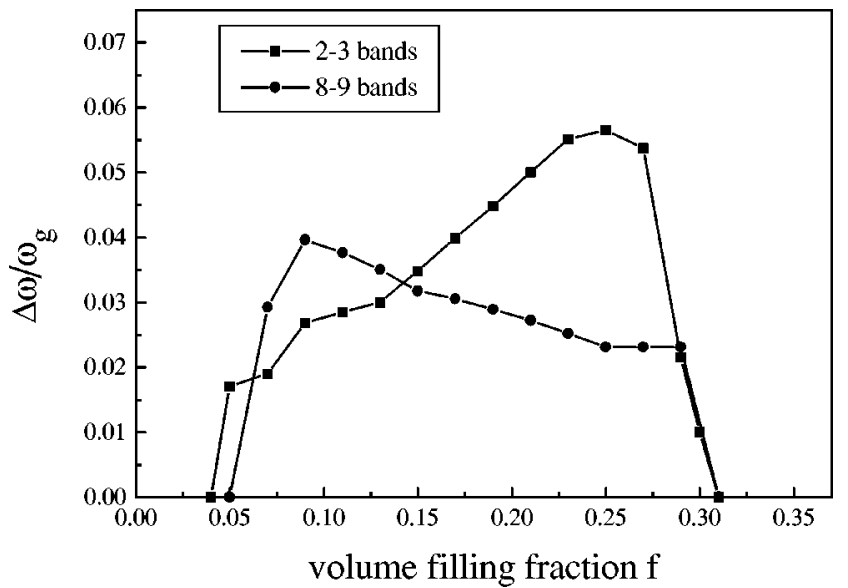

FIG. 7. Plots of $\Delta \omega / \omega_{g}$ of 2-3 and 8-9 partial band gaps vs filling fraction of Te sphere for a sc lattice.

regimes as the partial band gap still opens at a very low filling fraction for both fcc and bcc lattices.

The self-arrangement of colloidal crystals provides one way to produce $3 \mathrm{D}$ photonic band structures in the micrometer- or submicrometer-size regimes. ${ }^{23-26}$ Colloidal suspensions of highly charged, monodisperse polymer spheres can exhibit crystalline translational ordering in appropriate conditions of charge, number density, counterion concentration, and temperature. These colloidal suspensions can be crystallized into the fcc or bcc lattice at different volume fractions. However, such colloidal crystals do not exhibit a true photonic band gap because of relatively low index contrast. Then it is expected that the combination of such polymer material with the above mentioned anisotropic material with large principal indices and sufficient anisotropy between them will be likely to produce fcc or bcc structures, and will generate a partial band gap. Because of large refractive index, the anisotropic sphere coated with a thin polymer film of a low refractive index will have a photonic band structure close to that of a bare sphere. As the polymer thin film only holds a relatively low volume ratio of sphere, and it has a relatively low refractive index (about 1.5) compared with the material Te (with an average refractive index over 5.0), the Fourier transform coefficients of the dielectricity $\epsilon^{-1}(\mathbf{G})$ in Eq. (5) will alter a little when the Te sphere is coated with a thin polymer film. Then according to Eq. (7) for the dispersion relation of EM waves, the photonic band structures will also only change a little. Thus the polymercoated anisotropic PBG structure will still produce a partial band gap, similar to the uncoated PBG structure. Therefore this combination may provide a possible way to fabricate the $3 \mathrm{D}$ photonic crystal in the micrometer- and submicrometersize regimes.

Thus, uniaxial material Te may be chosen as a candidate material to produce 3D photonic crystal with a partial band gap in the infrared regime ( $\mathrm{Te}$ is a uniaxial crystal with $n_{e}$ $=6.2$ and $n_{o}=4.8$ in the wavelength regimes between $3.5 \mu \mathrm{m}$ and $35 \mu \mathrm{m}$ ). Although in the above sections we only consider the particular uniaxial material Te in the investigation of the anisotropic photonic band structures, the basic concepts are also applicable to other anisotropic materials with large principal refractive indices and sufficient anisotropy between them. Following these concepts one can search 
for an optimal anisotropic material for a PBG structure in various frequency regions of EM waves.

There remains one problem concerned with how to bring into the alignment the extraordinary axis of all the $\mathrm{Te}$ spheres in the same direction. In reality, it is difficult to obtain an exactly periodic photonic structure. However, because of sufficient anisotropy between the ordinary and extraordinary refractive indices, the application of a strong electric field may overcome this difficulty to some extent. Under the application of a strong field, the sphere may be aligned with its highest refractive-index axis along the electric field, as this configuration of spheres is most favorable thermodynamically. The free energy of the system reads $F$ $=-\Sigma_{i} \mathbf{p}_{i} \cdot \mathbf{E}_{i}$, where $\mathbf{p}_{i}$ is the electric dipole of a sphere, $\mathbf{E}_{i}$ is the local electric field. It is evident that when the highest refractive-index axis is along the applied field, the dipole $\mathbf{p}_{i}$ will reach its maximum in magnitude; thus the free energy is lowest for the configuration with the highest refractive-index axis (extraordinary axis) of all the uniaxial $\mathrm{Te}$ spheres aligned along the applied strong field. In this way, the thermodynamically favorable anisotropic PBG structure can be formed. Of course, disorder and defects due to thermodynamic instability in such a PBG structure may affect the character of its photonic band structure. This problem needs further investigation either theoretically or experimentally.

The PBG structure with a partial band gap will have some applications such as filters because it can still forbid the propagation of light in a wide range of solid angle with respect to the extraordinary axis. This is superior to $2 \mathrm{D}$ PBG structure, which is composed of periodic arrays of dielectric column in background medium. Although it is much easier to fabricate a 2D PBG structure in optical regimes, ${ }^{29,30}$ such a structure exhibits a full band gap only for light propagating perpendicular to the axis of the column. ${ }^{31-35}$ Light incident slantedly on the 2D PBG structure will not be totally reflected.

\section{SUMMARY AND CONCLUSIONS}

We have studied the photonic band structure composed of anisotropic dielectric spheres in the background of uniform dielectric medium for various lattices such as the diamond, fcc, bcc, and sc lattices. We solve Maxwell's equations for periodic anisotropic dielectric structures using the planewave expansion method. We find that for a uniaxial material with large principal refractive indices and sufficient anisotropy between them, the photonic band structures possess a full band gap in the whole Brillouin zone for the diamond lattice. Furthermore, in the $1 / 3$ partial Brillouin zone where the Bloch wave vector has a dominant component along the extraordinary axis of uniaxial material, the photonic-band structures exhibit partial band gaps for all the other simple lattices such as fcc, bcc, and sc lattices, although a complete band gap cannot be created in the whole Brillouin zone. The partial band gap can still be formed at a very low filling fraction of the uniaxial spheres. This phenomenon is attributed to the breakdown of the symmetry-induced band degeneracy and sufficient band splitting owing to anisotropy of material dielectricity. The combination of such an anisotropic PBG structure with the self-arrangement technique of colloidal crystal may provide a potential way to fabricate 3D photonic crystal in the visible and infrared regimes. Such anisotropic PBG structures will be of relevance in some applications because they can forbid the propagation of light in a wide range of solid angle with respect to the extraordinary axis of the uniaxial sphere. Further theoretical investigations and many experimental efforts are needed to bring the anisotropic photonic crystal into reality.

\section{ACKNOWLEDGMENTS}

The authors acknowledge the financial support of a CRCG grant from the University of Hong Kong (HKU), a RGC grant from the SAR Government of Hong Kong under Grant No. HKU 7112/97P, and a research grant from the National Natural Science Foundation of China. We also thank the computer center of HKU for computational facilities.
${ }^{1}$ E. Yablonovitch, Phys. Rev. Lett. 58, 2059 (1987).

2 S. John, Phys. Rev. Lett. 58, 2486 (1987).

${ }^{3}$ S. John and J. Wang, Phys. Rev. Lett. 64, 2418 (1990); Phys. Rev. B 43, 12772 (1991).

${ }^{4}$ S. John and T. Quang, Phys. Rev. A 50, 1764 (1994).

${ }^{5}$ J. Wang, Phys. Lett. A 204, 54 (1995).

${ }^{6}$ P. R. Villeneuve, S. Fan, and J. D. Joannopoulos, Phys. Rev. B 54, 7837 (1996).

${ }^{7}$ S. John and V. I. Rupasov, Phys. Rev. Lett. 79, 821 (1997).

${ }^{8}$ S. Y. Zhu, H. Chen, and H. Huang, Phys. Rev. Lett. 79, 205 (1997).

${ }^{9}$ For a review, see J. Opt. Soc. Am. B 10, 208 (1993), special issue on development and applications of materials exhibiting photonic band gaps.

${ }^{10}$ See Photonic Band Gaps and Localization, Proceedings of the NATO ARW, edited by C. M. Soukoulis (Plenum, New York, 1993).
${ }^{11}$ J. D. Joannopoulos, P. R. Villeneuve, and S. Fan, Nature (London) 386, 143 (1997).

${ }^{12}$ K. M. Leung and Y. F. Liu, Phys. Rev. Lett. 65, 2646 (1990).

${ }^{13}$ Z. Zhang and S. Satpathy, Phys. Rev. Lett. 65, 2650 (1990).

${ }^{14}$ K. M. Ho, C. T. Chan, and C. M. Soukoulis, Phys. Rev. Lett. 65, 3152 (1990).

${ }^{15}$ E. Yablonovitch, T. J. Gmitter, and K. M. Leung, Phys. Rev. Lett. 67, 2295 (1991).

${ }^{16}$ H. S. Sözüer and J. W. Haus, J. Opt. Soc. Am. B 10, 296 (1993).

${ }^{17}$ K. M. Ho, C. T. Chan, C. M. Soukoulis, R. Biswas, and M. Sigalas, Solid State Commun. 89, 413 (1994).

${ }^{18}$ E. Özbay, E. Michel, G. Tuttel, R. Biswas, M. Sigalas, and K. M. Ho, Appl. Phys. Lett. 64, 2059 (1994).

${ }^{19}$ C. C. Cheng and A. Scherer, J. Vac. Sci. Technol. B 13, 2696 (1995).

${ }^{20}$ S. Fan, P. R. Villeneuve, R. D. Meade, and J. D. Joannopoulos, 
Appl. Phys. Lett. 65, 1466 (1994).

${ }^{21}$ M. Wada, Y. Doi, K. Inoue, J. W. Haus, and Z. Yuan, Appl. Phys. Lett. 70, 2966 (1997).

${ }^{22}$ I. H. H. Zabel and D. Stroud, Phys. Rev. B 48, 5004 (1993).

${ }^{23}$ N. A. Clark, A. J. Hurd, and B. J. Ackerson, Nature (London) 281, 57 (1979).

${ }^{24}$ P. Pieranski, Contemp. Phys. 24, 25 (1983).

${ }^{25}$ I. I Tarhan and G. H. Watson, Phys. Rev. Lett. 76, 315 (1996).

${ }^{26}$ R. D. Pradhan, J. A. Bloodgood, and G. H. Watson, Phys. Rev. B 55, 9503 (1997).

${ }^{27}$ Handbook of Optics, edited by M. Bass (McGraw-Hill, New York, 1995), Vol. II, Chap. 33.

${ }^{28}$ C. Kittel, Introduction to Solid State Physics (Wiley, New York, 1996).
${ }^{29}$ U. Grüning, V. Lehmann, S. Ottow, and K. Busch, Appl. Phys. Lett. 68, 747 (1996).

${ }^{30}$ T. Krauss, R. De La Rue, and S. Band, Nature (London) 383, 699 (1996).

${ }^{31}$ S. L. McCall, P. M. Platzman, R. Dalichaouch, D. Smith, and S. Schultz, Phys. Rev. Lett. 67, 2017 (1991).

${ }^{32}$ W. M. Robertson, G. Arjavalingam, R. D. Meade, K. D. Brommer, A. M. Rappe, and J. D. Joannopoulos, Phys. Rev. Lett. 68, 2023 (1992).

${ }^{33}$ R. D. Meade, K. D. Brommer, A. M. Rappe, and J. D. Joannopoulos, Appl. Phys. Lett. 61, 495 (1992).

${ }^{34}$ P. R. Villeneuve and M. Piché, Phys. Rev. B 46, 4969 (1992).

${ }^{35}$ D. Cassagne, C. Jouanin, and D. Bertho, Phys. Rev. B 53, 7134 (1996). 